\title{
Paclitaxel PPE Microspheres
}

National Cancer Institute

\section{Source}

National Cancer Institute. Paclitaxel PPE Microspheres. NCI Thesaurus. Code C2785.

A paclitaxel formulation containing paclitaxel incorporated in biodegradable polyphosphoester (PPE) polymer form with potential antineoplastic activity. Upon intraperitoneal delivery, paclitaxel PPE microspheres slowly and continuously dissolve and deliver paclitaxel to the tumor site, where it binds to tubulin and inhibits the dynamics of disassembly-assembly of microtubules. As a result, this formulation induces cell cycle arrest and leads to cell death. 